\title{
Spherical Rectangles
}

\author{
Alexandre Eremenko ${ }^{1}$ • Andrei Gabrielov ${ }^{1}$
}

Received: 24 January 2016 / Revised: 9 August 2016 / Accepted: 30 August 2016 /

Published online: 9 September 2016

(C) Institute for Mathematical Sciences (IMS), Stony Brook University, NY 2016

\begin{abstract}
We study spherical quadrilaterals whose angles are odd multiples of $\pi / 2$, and the equivalent accessory parameter problem for the Heun equation. We obtain a classification of these quadrilaterals up to isometry. For given angles, there are finitely many one-dimensional continuous families which we enumerate. In each family the conformal modulus is either bounded from above or bounded from below, but not both, and the numbers of families of these two types are equal. The results can be translated to classification of Heun's equations with real parameters, whose exponent differences are odd multiples of $1 / 2$, with unitary monodromy.
\end{abstract}

Keywords Surfaces of positive curvature - Accessory parameters · Heun's equation · Elliptic integrals · Belyi functions

Mathematics Subject Classification 34M03 $\cdot 30 \mathrm{C} 20 \cdot 35 \mathrm{~J} 91 \cdot 33 \mathrm{E} 05$

\section{Introduction}

A (marked) circular polygon is a closed disk $Q$ with marked boundary points $a_{0}, \ldots, a_{n-1}$, which are called corners, enumerated cyclically according to the positive orientation of $\partial Q$, equipped with a Riemannian metric of constant curvature 1 with conic singularities at the corners, and such that each side $\left(a_{j}, a_{j+1}\right)$ has con-

A. Eremenko: Supported by NSF Grant DMS-1361836. A. Gabrielov: Supported by NSF Grant DMS-1161629.

$\bowtie$ Andrei Gabrielov

gabriea@purdue.edu

1 Department of Mathematics, Purdue University, West Lafayette, IN 47907, USA 
stant geodesic curvature. Two such polygons $Q$ and $Q^{\prime}$ are congruent if there is an orientation-preserving isometry between them which sends each corner $a_{j}$ of $Q$ to the corner $a_{j}^{\prime}$ of $Q^{\prime}$.

Polygons with $n=2,3$ and 4 are called digons, triangles and quadrilaterals, respectively.

If each side has zero geodesic curvature then $Q$ is called a spherical polygon. At every corner $a_{j}$, an interior angle $\alpha_{j} \geq 0$ is defined, and in what follows we measure all angles in half-turns. So angle $\alpha$ means an angle of $\pi \alpha$ radians, in particular, "integer angle" is an integer multiple of $\pi$ radians. A circular quadrilateral $Q$ whose angles are odd multiples of $1 / 2$ is called a circular rectangle. If such a quadrilateral is spherical, it is called a spherical rectangle. In this paper we describe the set of spherical rectangles with prescribed angles.

As every surface of positive curvature 1 is locally isometric to a piece of the unit sphere, every circular polygon can be described in terms of the developing map $f$ : $Q \rightarrow \overline{\mathbf{C}}$ which is an analytic function on $Q \backslash\left\{a_{0}, \ldots, a_{n-1}\right\}$ mapping every side into a circle on the Riemann sphere. For spherical polygons these circles are geodesic (great circles). This function $f$ is a local homeomorphism at each point except the corners, and at a corner $a$ satisfies

$$
f(z)-f(a) \sim c(z-a)^{\alpha},
$$

where $\alpha>0$ is the angle at this corner. (If $\alpha=0$, the right-hand side has to be replaced by $c / \log (z-a)$.)

Each such function defines a circular polygon by the pull-back of the spherical metric from $\overline{\mathbf{C}}$ to $Q$. If none of the $\alpha_{j}$ equals 1 , then the pair $\left(f, a_{0}\right)$ defines the polygon uniquely. Two pairs $\left(f_{1}, a_{0}\right)$ and $\left(f_{2}, a_{0}^{\prime}\right)$ define congruent polygons if $f_{2}=\psi \circ f_{1} \circ \phi$, where $\psi$ is a rotation of the Riemann sphere, and $\phi$ a conformal automorphism of the disk with the property $\phi\left(a_{j}\right)=a_{j}^{\prime}$ for all $j$.

This paper is a part of the project whose goal is to understand metrics of constant positive curvature with conic singularities on compact surfaces, see Troyanov (1991), Luo and Tian (1992), Eremenko (2004), Eremenko et al. (2014, 2016a, b), Mondello and Panov (2016), Lin and Wang (2010), Chai et al. (2015). An important class of such metrics can be obtained by gluing a spherical polygon to its mirror image isometrically along the boundary. Metrics of positive curvature on the sphere obtained in this way are characterized by the symmetry property: all conic singularities belong to a circle on the sphere, and the metric is symmetric with respect to this circle.

In this paper, we classify spherical rectangles. The cases when at least one of the angles of a spherical quadrilateral is integer were considered in Eremenko et al. (2006, 2014, 2016a, b).

If we use the upper half-plane as $Q$, then the developing map of every circular quadrilateral is a ratio of two linearly independent solutions of the Heun equation

$$
y^{\prime \prime}+\left(\sum_{j=0}^{2} \frac{1-\alpha_{j}}{z-a_{j}}\right) y^{\prime}+\frac{\alpha^{\prime} \alpha^{\prime \prime} z-\lambda}{\prod_{j=0}^{2}\left(z-a_{j}\right)} y=0,
$$


where

$$
\begin{aligned}
\alpha^{\prime} & =\left(2+\alpha_{3}-\alpha_{2}-\alpha_{1}-\alpha_{0}\right) / 2, \\
\alpha^{\prime \prime} & =\left(2-\alpha_{3}-\alpha_{2}-\alpha_{1}-\alpha_{0}\right) / 2,
\end{aligned}
$$

with the standard normalization $\left(a_{0}, a_{1}, a_{2}, a_{3}\right)=(0,1, a, \infty), a \in(1,+\infty)$. Here $\alpha_{j}$ are the angles at the corners, and $\lambda$ is a real accessory parameter. Each pair of linearly independent solutions of such an equation defines a circular quadrilateral. Different pairs of linearly independent solutions of the same equation define equivalent quadrilaterals: their developing maps are related by post-composition with linearfractional transformations. We will later see that an equivalence class may contain at most one spherical quadrilateral, up to congruence.

The condition that an equivalence class contains a spherical quadrilateral translates to the following condition on the Heun equation: the projective monodromy group must be conjugate to a subgroup of $S U$ (2). So the problem of classification of spherical quadrilaterals with prescribed corners and angles is equivalent to the problem of classification of Heun's equation with prescribed $a_{j}$ and $\alpha_{j}$ whose monodromy is unitarizable, that is conjugate to a subgroup of $S U(2)$. The correspondence between the metrics on the sphere and Heun's equations with unitarizable monodromy is bijective. Each symmetric metric on the sphere corresponds to two spherical quadrilaterals which are related by an anti-conformal involution, and to a unique normalized Heun equation with real $a, \lambda$ and unitarizable monodromy.

In the case that all angles are odd multiples of $1 / 2$, Heun's equation can be explicitly solved in terms of elliptic integrals. This fact was discovered by Darboux (1882) who generalized Hermite's work Hermite (1912) for the Lamé equation. For the study of general circular rectangles in connection with Heun's equation with $\alpha_{j}$ multiples of $1 / 2$ we refer to the paper of Van Vleck (1902).

We recall how this explicit solution is obtained.

Theorem A Suppose that all $\alpha_{j}$ in (1.1)-(1.3) are odd multiples of $1 / 2$. Then there are two linearly independent solutions of (1.1) whose ratio is of the form

$$
f(z)=\exp (I(z))
$$

where

$$
I(z)=\int_{z 0}^{z} \prod_{j=0}^{2}\left(\zeta-a_{j}\right)^{\alpha_{j}-1} \frac{d \zeta}{P(\zeta)}
$$

and $P(z)=P(z ; a, \lambda)$ is a real polynomial in all three variables. This polynomial satisfies the third order linear differential equation

$$
P^{\prime \prime \prime}+3 p P^{\prime \prime}+\left(p^{\prime}+2 p^{2}+4 q\right) P^{\prime}+\left(4 p q+2 q^{\prime}\right) P=0,
$$

where $p$ and $q$ are the coefficients in front of $y^{\prime}$ and $y$ in (1.1). 
Equation (1.6) has one-dimensional space of polynomial solutions of degree

$$
\operatorname{deg} P=\sum_{j=0}^{3} \alpha_{j}-2
$$

This permits to find $P$ by rational operations. That $P$ satisfies (1.6) guarantees that all residues of the integrand in (1.5) are of the form $\pm c$ with some real $c$. The condition $c=1$ defines $P$ up to a sign.

Periods of the integral (1.5), other than those coming from the residues, form a lattice generated by two canonical periods (integrals over adjacent real segments). Of these two canonical periods one is real and another is pure imaginary. The condition that the monodromy of (1.1) is unitarizable means that both periods must be imaginary, therefore the real period must vanish. For a fixed real $a$, and given angles, this imposes a transcendental equation on $\lambda$. It is not clear how to determine or estimate the number of real solutions of this equation, but for small angles $\alpha_{k}$ it can be solved numerically. The results of computation are described in Sect. 4.

Instead we use a geometric method which allows us to classify spherical rectangles, and describe their geometry. The following elementary statement was proved in Eremenko and Gabrielov (2015).

Proposition 1.1 Let $f$ be the developing map of a spherical rectangle $Q$. Then there are two opposite sides of $Q$ whose $f$-images are contained in the same circle, and the other pair of opposite sides is mapped to distinct circles.

Thus the boundary of a spherical rectangle $Q$ is mapped by $f$ to the union of three great circles, one of them, say $C$, being orthogonal to the other two, $C^{\prime}$ and $C^{\prime \prime}$. Let $\theta \in(0,1)$ be the angle between the circles $C^{\prime}$ and $C^{\prime \prime}$. There are two choices for this angle (the other one being $1-\theta$ ). See Definition 3.4 below for the unique choice of the angle $\theta$ associated with a spherical rectangle $Q$.

The $f$-preimage in $Q$ of the three circles is called the net of $Q$. The net is a combinatorial invariant of $Q$, defined up to an orientation-preserving homeomorphism of $Q$ respecting its initial corner $a_{0}$.

Proposition 1.2 A marked spherical rectangle $Q$ is defined uniquely up to isometry by its net and the angle $\theta \in(0,1)$.

This will be proved in Sect. 3. In Sect. 3, we will explicitly describe all possible nets of spherical rectangles (Theorems 3.1 and 3.5). As a consequence we will obtain the following necessary and sufficient conditions on the angles of a spherical rectangle:

Theorem 1.3 Let $A_{0}, \ldots, A_{3}$ be non-negative integers, and

$$
\delta=\left(A_{1}+A_{3}-A_{0}-A_{2}\right) / 2 .
$$

Then for the existence of a spherical rectangle with angles $\alpha_{j}=A_{j}+1 / 2$ it is necessary and sufficient that one of the following conditions be satisfied: either

$$
\delta \geq 1, \quad A_{1} \geq 1, \quad A_{3} \geq 1,
$$


or

$$
\delta \leq-1, \quad A_{0} \geq 1, \quad A_{2} \geq 1
$$

To state our next result we need some definitions. We recall that we consider marked spherical rectangles. Two of them are congruent if there is an orientation-preserving isometry of $Q$ respecting the initial corner $a_{0}$.

Every quadrilateral can be mapped conformally onto a flat rectangle with vertices $(0,1,1+i K, i K)$ and all angles $1 / 2$, such that $a_{0}$ maps to 0 . The number $K$ is called the modulus of the quadrilateral.

Each pair $(\Gamma, \theta)$, where $\Gamma$ is a net and $\theta \in(0,1)$, defines a marked spherical rectangle $Q(\Gamma, \theta)$ (see Theorem 3.5). Thus the set of all spherical rectangles with given angles consists of curves $\theta \mapsto Q(\Gamma, \theta)$ parameterized by $\theta$ and labeled by the nets. The modulus $K$ of $Q(\Gamma, \theta)$ is a continuous function of $\theta$. There are two kinds of these curves:

On the curves of the first kind, $K \rightarrow 0$ as $\theta \rightarrow 0$, while $K$ tends to a non-zero limit $K_{\text {crit }}(\Gamma)$ as $\theta \rightarrow 1$.

On the curves of the second kind, $K \rightarrow+\infty$ as $\theta \rightarrow 0$, while $K$ tends to a non-zero limit $K_{\text {crit }}(\Gamma)$ as $\theta \rightarrow 1$.

This is proved in Theorem 4.2, and we give few examples of computation of the limits $K_{\text {crit }}$ in Sect. 4. In all our examples $K$ is a monotone function of $\theta$. This is proved in Eremenko and Gabrielov (2015) for the simplest family of spherical quadrilaterals with angles $(3 / 2,1 / 2,3 / 2,1 / 2)$ but it is unlikely that this property holds in general. However, it is true for sufficiently small and large values of $K$.

Proposition 1.4 Each curve $Q(\Gamma, \theta)$ has finitely many intervals on which $K$ is monotone. In particular, for sufficiently small (resp., large) $K>0$, there is a unique spherical rectangle with the modulus $K$ in a curve $Q(\Gamma, \theta)$ of the first (resp., second) kind.

This follows from the general theory of o-minimal structures (see, e.g., van den Dries 1998), since the integral in (1.5) is a Pfaffian function in the sense of Khovanskii (1980). It was shown in Speissegger (1999) (see also Wilkie 1999) that the structure generated by Pfaffian functions is o-minimal.

Our final results count the nets for spherical rectangles with given angles.

The quadruple $\left(A_{0}, \ldots, A_{3}\right)$ is special if $\delta$ in (1.8) is an odd integer and one of the following holds: either $A_{1} \geq \delta>0$ and $A_{3} \geq \delta>0$ or $A_{0} \geq-\delta>0$ and $A_{2} \geq-\delta>0$.

Define

$$
\begin{gathered}
M_{1}=\left[\min \left\{\frac{A_{1}+1}{2}, \frac{A_{3}+1}{2}, \frac{1+\delta}{2}\right\}\right], \\
M_{2}=\left[\min \left\{\frac{A_{0}+1}{2}, \frac{A_{2}+1}{2}, \frac{1-\delta}{2}\right\}\right], \\
N=\min \left\{A_{0}+\frac{1+\delta}{2}, A_{1}+\frac{1-\delta}{2}, A_{2}+\frac{1+\delta}{2}, A_{3}+\frac{1-\delta}{2}\right\} .
\end{gathered}
$$


Note that conditions (1.9) and (1.10) are satisfied when $M_{1}>0$ and $M_{2}>0$, respectively.

Theorem 1.5 For a special quadruple $\left(A_{0}, \ldots, A_{3}\right)$ satisfying either (1.9) or (1.10) there exist $2 N$ one-parametric families of congruence classes of marked spherical rectangles with angles $A_{j}+1 / 2$. If $\left(A_{0}, \ldots, A_{3}\right)$ is not special but satisfies (1.9) (resp., (1.10)) then there exist $2 M_{1}$ (resp., 2M $M_{2}$ ) one-parametric families of congruence classes of marked spherical rectangles with angles $A_{j}+1 / 2$.

Each family is parameterized by $\theta \in(0,1)$ (see Definition 3.4). Each family contains either rectangles of arbitrarily small moduli or arbitrarily large moduli but not both. The numbers of families of both types are equal, so for each type this number is either $N$ or $M_{1}$ or $M_{2}$, depending on $\left(A_{0}, \ldots, A_{3}\right)$.

Remark 1.6 Theorem 1.5 and Proposition 1.4 imply that the number of spherical rectangles with given angles $A_{j}+1 / 2$ is exactly $N$ or $M_{1}$ or $M_{2}$, depending on $\left(A_{0}, \ldots, A_{3}\right)$, for sufficiently small and large values of $K$.

For fixed angles $\alpha_{j} \in \mathbf{N}^{+}+1 / 2$, consider the two-parametric family of Heun's equations (1.1) with parameters $(a, \lambda) \in(1,+\infty) \times \mathbf{R}$. Equivalence classes of circular rectangles are in correspondence to such Heun's equations. One-parametric families of spherical rectangles of Theorem 1.5 correspond to smooth disjoint curves in the half-plane $(a, \lambda)$, each having one end in this half-plane. On the other end, either $a \rightarrow 1$ or $a \rightarrow \infty$.

When $\theta=p / q$ is rational, the monodromy group of the developing map is finite, so $f$ is an algebraic function. In this case, $f=g^{-1} \circ h$, where

$$
g=-\frac{1}{4}\left(z^{q}+\frac{1}{z^{q}}-2\right)
$$

and $h$ is a rational Belyi function, which means that the only critical values of $h$ are $0,1, \infty$, Schneps (1994). Function $g$ is also a Belyi function, it is called the fundamental rational function of the dihedral group Klein (1993). The set $g^{-1}(\mathbf{R})$ consists of the unit circle and $q$ lines $\{z=t \exp (\pi i k / q): t \in \mathbf{R}, k=0, \ldots, q-1\}$. In the simplest case $\theta=1 / 2$, the image $f(\partial Q)$ is contained in the union of the unit circle $C$, real line $C^{\prime}$, and imaginary line $C^{\prime \prime}$. The monodromy is the Klein Viergroup $\mathbf{Z}^{2} \times \mathbf{Z}^{2}$, represented as $\{z,-z, 1 / z,-1 / z\}$ and $g$ has the property that $g^{-1}(\mathbf{R})=C \cup C^{\prime} \cup C^{\prime \prime}$. Then it is easy to see that our net, together with its reflection in the real line, coincides with $h^{-1}(\mathbf{R})$. The set $h^{-1}(\mathbf{R})$ for a Belyi function $h$ is a triangulation of the sphere with all vertices of even degree. Therefore our classification of the nets can be restated as classification of triangulations $\mathbf{T}$ of the sphere with the following properties:

(a) $\mathbf{T}$ is symmetric with respect to $\mathbf{R}$, and $\mathbf{R}$ is contained in the 1-skeleton of $\mathbf{T}$,

(b) There are four vertices $a_{j}$ of $\mathbf{T}$ on the real line of prescribed orders $4 A_{j}+2$.

(c) All other vertices of $\mathbf{T}$ have order 4 .

For $q \in\{2,3\}$ and $\left(A_{0}, \ldots, A_{3}\right)=(1,0,1,0)$, algebraic developing maps are explicitly written in Eremenko and Gabrielov (2015). 


\section{Spherical Polygons with the Sides on Three Circles and Corners at Their Intersections}

In this section we prove a preliminary result for classification of nets. Roughly speaking it says that every spherical rectangle is a union of two spherical triangles.

As we prove this result by induction, it is convenient to consider a more general class of spherical polygons, characterized by the property that the developing map sends their sides to three transversally intersecting great circles and corners to the intersection points of these circles. The net $\Gamma$ defines a triangulation of such a polygon $Q$, each face of it being mapped by $f$ one-to-one onto one of the triangles into which the three circles partition the sphere. This triangulation satisfies the following properties:

(P1) Each vertex inside $Q$ has degree 4;

(P2) All boundary vertices, other than corners of $Q$, have degree 3.

Combining this triangulation with its mirror copy, we obtain a triangulation $\mathbf{T}$ of the sphere satisfying the following properties:

(S1) $\mathbf{T}$ is symmetric with respect to a circle $S$ contained in the 1-skeleton of $\mathbf{T}$;

(S2) Each vertex of $\mathbf{T}$ has even degree, and all its vertices not contained in $S$ have degree 4.

It is easy to show that the nets of spherical polygons with all sides mapped to three transversal circles and all corners to intersection points of those circles are in one-toone correspondence with triangulations of the sphere satisfying (S1) and (S2).

Two nets are combinatorially equivalent if they can be obtained from each other by an orientation-preserving homeomorphism (mapping corners to corners and sides to sides) preserving the initial corner.

If $C$ is any of the three circles, its preimage in $Q$ is called $C$-net, denoted $\Gamma_{C}$. An $\operatorname{arc}$ of the net $\Gamma_{C}$ (or an arc of $\Gamma$ if $C$ is not specified) is a connected component of $\Gamma_{C} \backslash \partial Q$. Since $f$ is a local homeomorphism on the interior of $Q$, an arc may be homeomorphic to either an open interval with both ends on the boundary of $Q$ (possibly, at the same corner of $Q$ ) or a circle in the interior of $Q$. We'll show below (see Corollary 2.3) that an arc of a spherical rectangle $Q$ must have at least one end at a corner of $Q$. In particular, an arc of a spherical rectangle cannot be a circle. An arc is called short if it does not intersect other arcs of $\Gamma$. Any two arcs of the same net $\Gamma_{C}$ are disjoint.

Definition 2.1 We say that a spherical polygon $Q$ is reducible if its net has an arc with endpoints at two distinct corners of $Q$. Such an arc partitions $Q$ into two proper subpolygons. Otherwise, $Q$ is irreducible. We say that $Q$ is primitive if it is irreducible and its net does not contain an arc with both ends at the same corner of $Q$.

Theorem 2.2 Let $Q$ be a spherical $n$-gon such that all its sides are mapped to three transversal great circles by the developing map, and all its corners are mapped to intersection points of those circles. Then either $n \leq 3$ or there is a triangulation of $Q$ by $n-3$ disjoint arcs of its net, each of them connecting two non-adjacent corners of $Q$.

Proof It is enough to show that, unless $Q$ is a digon or a triangle, there exists an arc of its net $\Gamma$ connecting two of its non-adjacent corners. We prove this by induction on 
the number $N$ of faces of $\Gamma$. If $\Gamma$ has one face then, since any face of $\Gamma$ is a triangle, $Q$ is a triangle.

If $N>1$ then there exists an $\operatorname{arc} \gamma$ of $\Gamma$ adjacent to a point $p$ on the boundary of $Q$. Otherwise the face of $\Gamma$ adjacent to its boundary would not be simply connected.

If $\gamma$ connects two distinct corners $p$ and $q$ of $Q$ then either $p$ and $q$ are non-adjacent and we are done, or $p$ and $q$ are adjacent corners of $Q$, and $\gamma$ partitions $Q$ into a digon and a polygon $Q^{\prime}$ with the same number of corners as $Q$ and a smaller than $N$ number of faces of its net. By inductive hypothesis, unless $Q^{\prime}$ (and thus $Q$ ) is a digon or a triangle, there is an arc $\gamma^{\prime}$ of $Q^{\prime}$ connecting two of its non-adjacent corners. In the latter case, $\gamma^{\prime}$ is also an arc of $\Gamma$ connecting two non-adjacent corners of $Q$, and we are done.

Suppose now that $\Gamma$ does not have any arcs connecting two corners of $Q$. If $\gamma$ has both ends at the same point $p$ then $Q$ can be replaced by a $(n+1)$-gon $Q^{\prime}$ having all sides of $Q$ plus $\gamma$ as its sides, with the number of faces of the net $\Gamma^{\prime}$ of $Q^{\prime}$ smaller than $N$. There is a mapping $\iota: Q^{\prime} \rightarrow Q$ such that any two distinct points of $Q^{\prime}$ map to distinct points of $Q$, except the two ends of the side $\gamma$ of $Q^{\prime}$ that both map to $p$. By the inductive hypothesis, there is an arc $\gamma^{\prime}$ of $\Gamma^{\prime}$ connecting two non-adjacent corners $p^{\prime}$ and $q^{\prime}$ of $Q^{\prime}$. Then $\iota\left(\gamma^{\prime}\right)$ is an arc of $\Gamma$ connecting two (possibly, adjacent) corners of $Q$, a contradiction.

Thus we may suppose that $\gamma$ has two distinct ends $p$ and $q$ on the boundary of $Q$, at least one of them not a corner of $Q$. Then $\gamma$ partitions $Q$ into two polygons $Q^{\prime}$ and $Q^{\prime \prime}$, with the number of corners $n^{\prime}$ and $n^{\prime \prime}$ respectively, where $n^{\prime}+n^{\prime \prime} \geq n+3$. If $n>3$ then at least one of $n^{\prime}$ and $n^{\prime \prime}$ is greater than 3. Since both $Q^{\prime}$ and $Q^{\prime \prime}$ have the number of faces of their nets smaller than $N$, by the inductive hypothesis at least one of them has an arc $\gamma^{\prime}$ of its net connecting two non-adjacent corners. Then $\gamma^{\prime}$ is also an arc of $\Gamma$ connecting two non-adjacent corners of $Q$. This completes the proof.

Corollary 2.3 If $Q$ is a spherical $n$-gon with $n \geq 3$ satisfying the condition of Theorem 2.2 then an arc $\gamma$ of the net of $Q$ is an open interval with at least one end at a corner of $Q$.

Proof It follows from classification of spherical triangles (see sections 10 and 12 of Eremenko et al. 2016a, section 6 of Eremenko et al. 2016b and Figs. 1, 2 and 3a) that an arc of the net of a spherical triangle with all sides mapped to three circles $C, C^{\prime}, C^{\prime \prime}$ and all corners to intersection points of those circles must have at least one end at its corner. Indeed, such a triangle $T$ contains a primitive triangle $T^{\prime}$ (either one of triangles $T_{\mu}$ or one of triangles $E_{\mu}$, see Fig. 1) with its apex at the intersection of two circles, say $C$ and $C^{\prime}$, and its base on the third circle $C^{\prime \prime}$. Any arc of the net of $T^{\prime}$ connects its apex with its base. The triangle $T$ is obtained by attaching digons to the sides of $T^{\prime}$ (see Fig. 3a). An arc of the net of a digon $D$ either has an end at one of its corners or the ends on both its sides, but cannot have both ends on one side of $D$. Thus an arc of $T$ must have at least one end at its corner. Since the intersection of $\gamma$ with any triangle $T$ of a triangulation of $Q$ by disjoint arcs of its net connecting its non-adjacent corners is either a side of $T$ or an $\operatorname{arc}$ of the net of $T$, it must have at least one end at a corner of $T$. But all corners of $T$ are also corners of $Q$. 

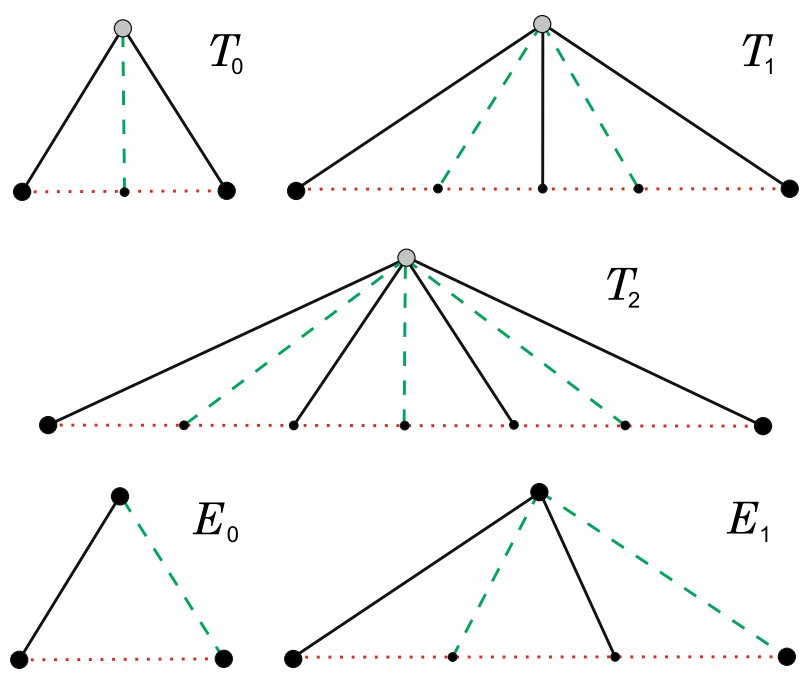

Fig. 1 Primitive spherical triangles $T_{\mu}$ and $E_{\mu}$

$D_{1}$

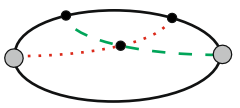

$D_{1}$

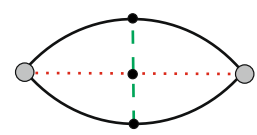

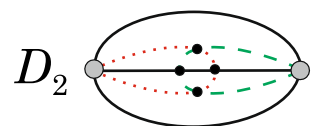
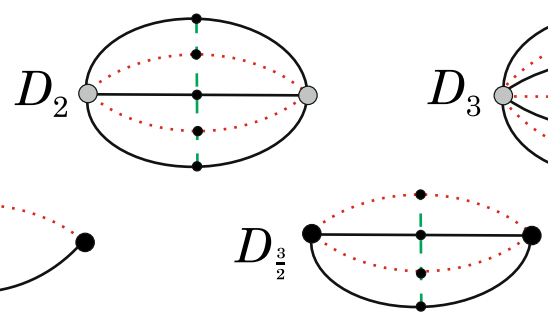
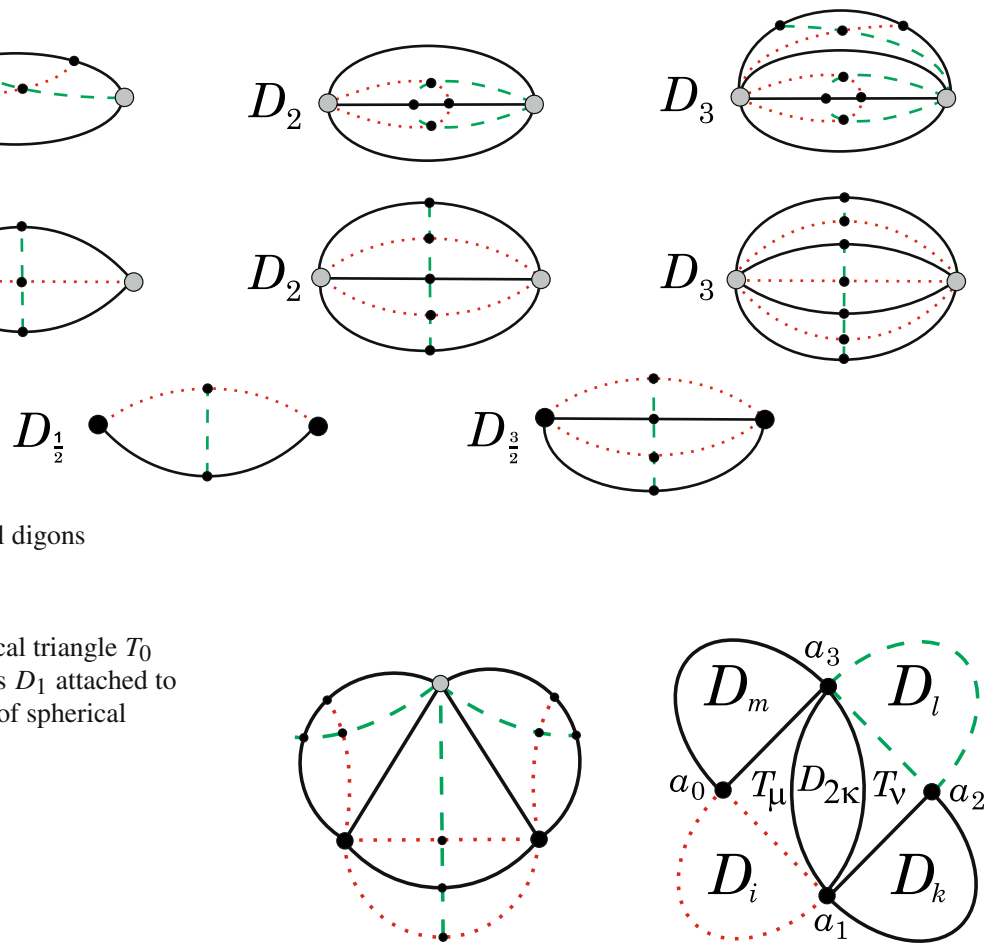

(b) 

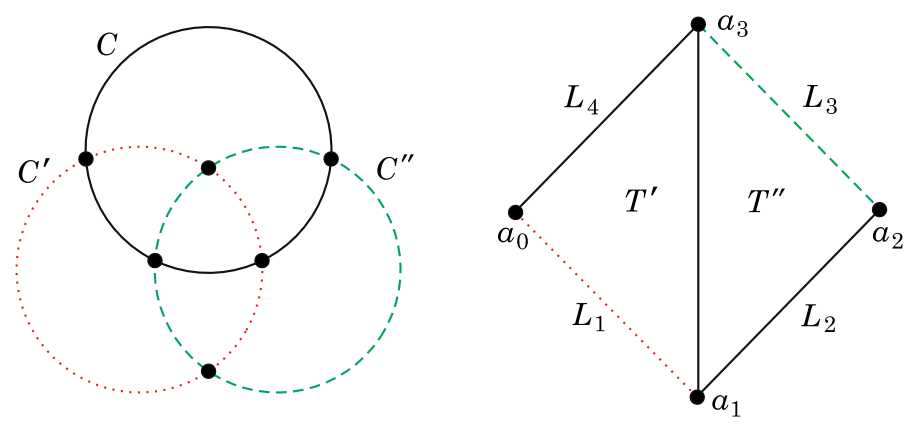

Fig. 4 Three circles (left) and a spherical rectangle (right)

\section{Nets of Spherical Rectangles}

As was shown in Eremenko and Gabrielov (2015) (see Proposition 1.1) any spherical rectangle $Q$ has two opposite sides mapped to the same circle by its developing map $f$, and two other opposite sides mapped to distinct circles. Thus there are two types of marked spherical rectangles: in the first type the images of $L_{2}$ and $L_{4}$ belong to the same circle, and in the second type the images of $L_{1}$ and $L_{3}$ belong to the same circle. It is enough to classify spherical rectangles of the first type, as all rectangles of the second type can be obtained from those of the first type by orientation-reversing isometry preserving the marked corner.

Assumption 1 Unless stated otherwise, all spherical rectangles below are assumed to be of the first type.

Let $C$ be the circle to which two sides $L_{2}$ and $L_{4}$ of a spherical rectangle $Q$ are mapped, and let $C^{\prime}$ and $C^{\prime \prime}$ be the circles to which the sides $L_{1}$ and $L_{3}$ of $Q$ are mapped (see Fig. 4).

Theorem 2.2 implies that there is an arc of the net $\Gamma$ of $Q$ connecting two opposite corners of $Q$. Such an arc must be mapped to the circle $C$, since two opposite corners of $Q$ are mapped to intersection points of $C$ with two distinct circles other than $C$. This implies that $Q$ cannot have two arcs of $\Gamma$ connecting two pairs of its opposite corners: such arcs would have an intersection point inside $Q$, while any two arcs mapped to the same circle $C$ are disjoint.

Assumption 2 Unless stated otherwise, we choose the initial corner $a_{0}$ of a marked spherical rectangle $Q$ so that there is an arc $\gamma$ of $\Gamma$ connecting the corners $a_{1}$ and $a_{3}$ of $Q$.

Such an arc $\gamma$ partitions $Q$ into two spherical triangles $T^{\prime}$ and $T^{\prime \prime}$, where $T^{\prime}$ has an integer corner at $a_{3}$ and the base $L_{1}$ mapped to $C^{\prime}$, while $T^{\prime \prime}$ has an integer corner at $a_{1}$ and the base $L_{3}$ mapped to $C^{\prime \prime}$ (see Fig. 4). We'll show below (see Remark 3.2) that the angles of such rectangle $Q$ satisfy the inequality $A_{0}+A_{2}+2 \leq A_{1}+A_{3}$.

Any rectangle of the first (resp., second) type with an arc of its net connecting its corners $a_{0}$ and $a_{2}$ can be obtained from a rectangle of the second (resp, first) type with an arc of its net connecting its corners $a_{1}$ and $a_{3}$ by choosing $a_{1}$ instead of $a_{0}$ as 
an initial corner, and relabeling the corners accordingly. The angles of such rectangle satisfy the inequality $A_{1}+A_{3}+2 \leq A_{0}+A_{2}$. Thus it is enough to classify spherical rectangles satisfying Assumptions 1 and 2.

Theorem 3.1 Let $Q$ be a marked spherical rectangle satisfying Assumptions 1 and 2. Then $Q$ is a union of two primitive triangles $T_{\mu}$ and $T_{\nu}$ having integer angles $\mu+1$ and $v+1$, respectively, digon $D_{2 \kappa}$ with the sides mapped to $C$ having common sides with both $T_{\mu}$ and $T_{\nu}$, digon $D_{i}$ with the sides mapped to $C^{\prime}$ attached to the base of $T_{\mu}$, digon $D_{l}$ with the sides mapped to $C^{\prime \prime}$ attached to the base of $T_{v}$, digon $D_{m}$ attached to the remaining side of $T_{\mu}$, and digon $D_{k}$ attached to the remaining side of $T_{\nu}$. The sides of $D_{k}$ and $D_{m}$ are mapped to $C$.

Here $\mu, v, \kappa, i, k, l, m$ are non-negative integers satisfying $i \mu=l v=0$, that is, $i>0$ only if $\mu=0, l>0$ only if $v=0$. The value 0 for $i, k, l, m, \kappa$ means there is no digon attached.

Proof Assumptions 1 and 2 imply that an $\operatorname{arc} \gamma$ of the net $\Gamma$ of $Q$ connecting its corners $a_{1}$ and $a_{3}$ partitions $Q$ into two spherical triangles $T^{\prime}$ and $T^{\prime \prime}$, where $T^{\prime}$ has an integer corner with an angle $\mu+1$ at $a_{3}$, and $T^{\prime \prime}$ has an integer corner with an angle $v+1$ at $a_{1}$, for some non-negative integers $\mu$ and $\nu$.

Classification of spherical triangles with one integer corner (see sections 10 and 12 of Eremenko et al. 2016a) implies that the triangle $T^{\prime}$ (resp., $T^{\prime \prime}$ ) is combinatorially equivalent to a primitive triangle $T_{\mu}$ (resp., $T_{\nu}$ ) having an angle $\mu+1$ (resp., $v+1$ ) at its integer corner, with digons attached to its sides (see Figs. 1, 2, 3a). No digons may be attached to the base $L_{1}$ of $T^{\prime}$ (resp., the base $L_{3}$ of $T^{\prime \prime}$ ) if $\mu>0$ (resp., $v>0$ ). Each digon $D_{n}$ has equal integer angles $n$ at its two corners.

The sides of $T_{\mu}$ and $T_{\nu}$ are mapped to $C$ and cannot contain preimages of intersection points of $C$ with either $C^{\prime}$ or $C^{\prime \prime}$, other than the corners of $Q$. This implies that the union of digons attached to the sides of $T_{\mu}$ and $T_{v}$ and having $\gamma$ as their common side is a digon $D_{2 \kappa}$ with even integer angles $2 \kappa$ at its two corners.

Thus a net of a spherical rectangle satisfying Assumptions 1 and 2 must have the structure shown schematically in Fig. 3b. This proves Theorem 3.1.

Remark 3.2 The angles at the corners $a_{0}, a_{1}, a_{2}, a_{3}$ of a marked spherical rectangle $Q$ in Theorem 3.1 have the integer parts

$$
\begin{aligned}
& A_{0}=i+m, \quad A_{1}=i+k+v+1+2 \kappa, \quad A_{2}=k+l, \\
& A_{3}=l+m+\mu+1+2 \kappa,
\end{aligned}
$$

respectively. In particular, $A_{0}+A_{2}+2 \leq A_{1}+A_{3}$. For a marked spherical rectangle (of either first or second type) with an arc of its net connecting its corners $a_{0}$ and $a_{2}$, the integer parts of its angles satisfy $A_{1}+A_{3}+2 \leq A_{0}+A_{2}$.

Remark 3.3 Theorem 3.1 implies that a spherical rectangle $Q$ satisfying Assumptions 1 and 2 has at least one short arc $\gamma$ connecting its corners $a_{1}$ and $a_{3}$, and that all such short arcs are mapped to the same $\operatorname{arc} \beta$ of the circle $C$ with the ends at the intersection points of $C$ with $C^{\prime}$ and $C^{\prime \prime}$. 
Definition 3.4 The angle $\theta \in(0,1)$ between the circles $C^{\prime}$ and $C^{\prime \prime}$ is defined as $1-\alpha$ where $\alpha$ is the length of any short arc $\gamma$ of the net of a spherical rectangle $Q$ connecting its opposite corners, divided by $\pi$. Alternatively, $\alpha$ is the length of the $\operatorname{arc} \beta$ of $C$ to which $\gamma$ is mapped, divided by $\pi$.

Proof of Proposition 1.2 We want to show that two marked spherical rectangles $Q$ and $Q^{\prime}$ with equivalent nets $\Gamma$ and $\Gamma^{\prime}$, and the same angle $\theta$, are congruent, i.e., there is an orientation-preserving isometry $Q \rightarrow Q^{\prime}$ mapping the initial corner $a_{0}$ of $Q$ to the initial corner $a_{0}^{\prime}$ of $Q^{\prime}$. According to Definition 3.4, the developing map $f: Q \rightarrow \overline{\mathbf{C}}$ maps the sides of $Q$ to three great circles $C, C^{\prime}, C^{\prime \prime}$, such that both $C^{\prime}$ and $C^{\prime \prime}$ are orthogonal to $C$, so that the sides $L_{2}$ and $L_{4}$ of $Q$ are mapped to $C$, the sides $L_{1}$ and $L_{3}$ of $Q$ are mapped to $C^{\prime}$ and $C^{\prime \prime}$, respectively, and any short arc of $\Gamma$ connecting the corners $a_{1}$ and $a_{3}$ of $Q$ is mapped to an $\operatorname{arc} \beta$ of $C$ of length $\pi \alpha$ where $\alpha=1-\theta$, the ends $P$ and $R$ of $\beta$ being the images of $a_{1}$ and $a_{3}$, respectively. In particular, $P$ and $R$ are intersection points of $C$ with $C^{\prime}$ and $C^{\prime \prime}$, respectively. Similarly, the developing map $g: Q^{\prime} \rightarrow \overline{\mathbf{C}}$ maps the sides of $Q^{\prime}$ to three great circles $S, S^{\prime}, S^{\prime \prime}$, such that both $S^{\prime}$ and $S^{\prime \prime}$ are orthogonal to $S$, and any short arc $\gamma^{\prime}$ of $\Gamma^{\prime}$ connecting the corners $a_{1}^{\prime}$ and $a_{3}^{\prime}$ of $Q^{\prime}$ is mapped to an $\operatorname{arc} \beta^{\prime}$ of $S$ of length $\pi \alpha$, the ends $P^{\prime}$ and $R^{\prime}$ of $\beta^{\prime}$ being the images of $a_{1}^{\prime}$ and $a_{3}^{\prime}$, respectively. Applying if necessary rotation of $\overline{\mathbf{C}}$, we may assume that $S=C, S^{\prime}=C^{\prime}, S^{\prime \prime}=C^{\prime \prime}, \beta^{\prime}=\beta, P^{\prime}=P$, and $R^{\prime}=R$. Then the equivalence of the nets $\Gamma$ and $\Gamma^{\prime}$ implies that the developing maps $f$ and $g$ send the corresponding faces, edges and vertices of partitions of $Q$ and $Q^{\prime}$ to the same triangles, segments and intersection points of the partition of $\overline{\mathbf{C}}$ by the three circles $C, C^{\prime}$ and $C^{\prime \prime}$. In particular, $a_{0}$ and $a_{0}^{\prime}$ are mapped by $f$ and $g$ to the same point of $\overline{\mathbf{C}}$.

Theorem 3.5 For any non-negative integers $\mu, \nu, \kappa, i, k, l, m$ satisfying $i \mu=l v=0$ there is a unique, up to combinatorial equivalence, net $\Gamma$ of the type described in Theorem 3.1. For any such net $\Gamma$ and any $\theta \in(0,1)$ there exists a unique spherical rectangle $Q=Q(\Gamma, \theta)$ having $\Gamma$ as its net, sides mapped to three circles $C, C^{\prime}, C^{\prime \prime}$, and $a$ short arc of length $\pi(1-\theta)$ connecting its corners $a_{1}$ and $a_{3}$.

Proof To define the net $\Gamma$, we start with a digon $D_{2 \kappa}$ obtained by combining $\kappa$ copies of digon $D_{2}$ shown in the middle of the first row of Fig. 2. If $\kappa=0$ then there is no such digon, and we proceed with gluing together triangles $T_{\mu}$ and $T_{\nu}$ (see Fig. 1) so that the side of each of these triangles that follows its base in the counterclockwise cyclic order becomes their common side. If $\kappa>0$ then triangles $T_{\mu}$ and $T_{\nu}$ are attached to opposite sides of $D_{2 \kappa}$ so that the side of each of these triangles that follows its base in the counterclockwise cyclic order becomes its common side with $D_{2 \kappa}$. The integer corner of $T_{\mu}$ (resp., $T_{\nu}$ ) coincides with a non-integer corner of $T_{\nu}$ (resp., $T_{\mu}$ ).

Next, we attach digons $D_{k}$ and $D_{m}$, obtained by combining $k$ and $m$ copies, respectively, of the digon $D_{1}$ shown in the left side of the first row of Fig. 2, so that any two adjacent digons have either a common short side or a common long side, and so that each of the two resulting digons has at least one short side, as is shown in examples of $D_{2}$ and $D_{3}$ in the first row of Fig. 2. Then digons $D_{k}$ and $D_{m}$ are attached to the free sides of $T_{\nu}$ and $T_{\mu}$, respectively. The free sides of these triangles are preceding their respective bases in the counterclockwise cyclic order. If $k=0$ (resp., $m=0$ ) then no digon $D_{k}$ (resp., $D_{m}$ ) is attached. 
(a)

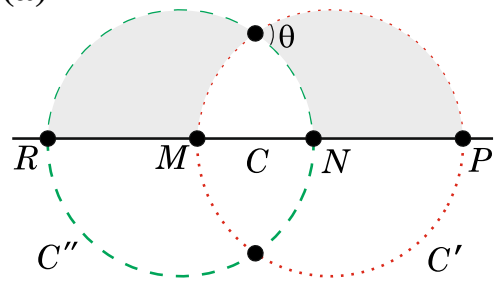

(c)

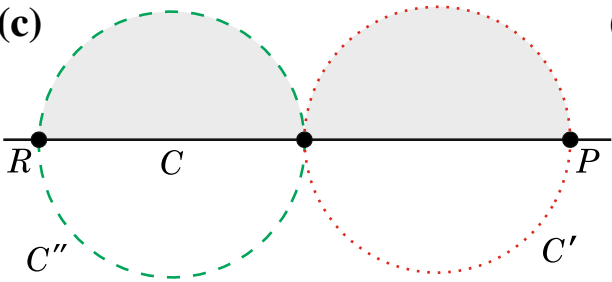

(b)

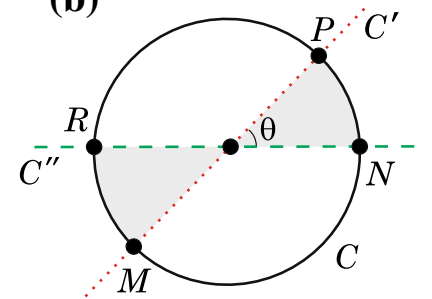

(d)

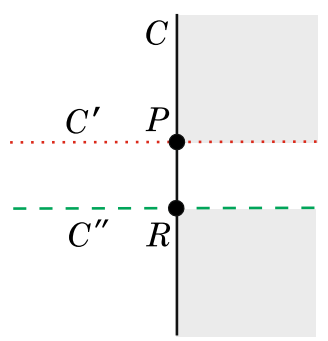

Fig. 5 Deformation of the three-circle configuration

Finally, if $\mu=0$ and $i>0$ (resp., $v=0$ and $l>0$ ) then a digon $D_{i}$ (resp., $D_{l}$ ), obtained by combining $i$ and $l$ copies, respectively, of the digon $D_{1}$ shown in the second row of Fig. 2, is attached to the base of $T_{\mu}$ (resp., $T_{\nu}$ ). Examples of such digons are shown in the second row of Fig. 2.

If we label the sides of $T_{\mu}$ and $T_{\nu}$ by $C$, the base of $T_{\mu}$ by $C^{\prime}$ and the base of $T_{\nu}$ by $C^{\prime \prime}$ then all edges of $\Gamma$ can be uniquely labeled so that the sides of each of its triangles are labeled by three distinct labels. Consider the standard sphere $\overline{\mathbf{C}}$ with three great circles $C, C^{\prime}$ and $C^{\prime \prime}$ such that $C$ is orthogonal to both $C^{\prime}$ and $C^{\prime \prime}$, and one of the two complementary angles between $C^{\prime}$ and $C^{\prime \prime}$ is $\theta$, the other one being $\alpha=1-\theta$. Then there is a mapping of $Q$ to $\overline{\mathbf{C}}$, locally one-to-one everywhere except at the corners, which is unique up to a homeomorphism of $Q$ preserving all vertices and edges of $\Gamma$ and a rotation of the sphere preserving the three circles, such that any arc of the boundary of $D_{2 \kappa}$ (or a common side of $T_{\mu}$ and $T_{\nu}$ if $\kappa=0$ ) maps to an $\operatorname{arc}$ of $C$ of length $\pi \alpha$, and each edge of $\Gamma$ maps to an arc of the circle corresponding to its label. This defines on $Q$ a metric of the spherical rectangle $Q(\Gamma, \theta)$.

\section{Limits at $\theta=0$ and $\theta=1$ of Spherical Rectangles with the Given Net}

Each admissible set of integers $\mu, v, \kappa, i, k, l, m$ in Theorem 3.1 defines a net $\Gamma$ and the corresponding one-parametric family of marked spherical rectangles $Q(\Gamma, \theta)$ satisfying Assumptions 1 and 2, parameterized by the angle $\theta$ between the circles $C^{\prime}$ and $C^{\prime \prime}$ (see Definition 3.4). These two circles intersect the circle $C$ at the right angle. In Fig. 5a, b, two projections of the three circles are shown, and two of the triangles of the partition of the sphere defined by these circles are shaded.

Let $P$ and $R$ be the images of the corners $a_{1}$ and $a_{3}$, respectively, of a marked spherical rectangle $Q$, so that the $\operatorname{arc} \beta=P R$ of $C$ is the image of a short $\operatorname{arc} \gamma$ of the 


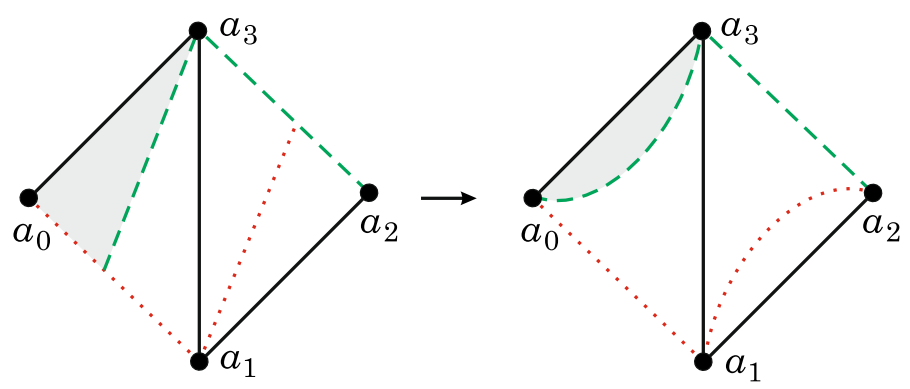

Fig. 6 Deformation of spherical rectangles with the angles $\frac{1}{2}, \frac{3}{2}, \frac{1}{2}, \frac{3}{2}$

net $\Gamma$ of $Q$ connecting $a_{1}$ and $a_{3}$. Then the shaded areas in Fig. 5a, b contract to arcs when $\theta \rightarrow 0$ and expand to half-disks when $\theta \rightarrow 1$. If all circles remain geodesic, then $C^{\prime}$ and $C^{\prime \prime}$ converge to the same circle when $\theta \rightarrow 0$ and when $\theta \rightarrow 1$. However, applying a linear-fractional transformation depending on $\theta$, so that the arc $M N$ of $C$ in Fig. 5a, b contracts to a point while the arc $P R$ does not, we can obtain in the limit $\theta \rightarrow 1$ a non-geodesic configuration shown in two different projections in Fig. 5c, d, where the shaded areas are the limits of the shaded areas in Fig. 5a, b.

Example 4.1 A net of a spherical rectangle $Q$ with the angles $(1 / 2,3 / 2,1 / 2,3 / 2)$ considered in Eremenko and Gabrielov (2015) is shown in Fig. 6 (left). The shaded area corresponds to preimage of the shaded areas in Fig. 5a, b. The arc connecting $a_{1}$ and $a_{3}$ is mapped to the arc $P R$ of $C$, the corners $a_{0}$ and $a_{2}$ are mapped to $M$ and $N$, respectively. When $\theta \rightarrow 0$, the sides $L_{2}$ and $L_{4}$ of $Q$ are contracted to points, while the distance between them has a positive limit. Thus the modulus of $Q$ has limit 0 as $\theta \rightarrow 0$ (see Eremenko et al. 2016a, Section 15, and Eremenko and Gabrielov 2015). When $\theta \rightarrow 1$, applying a linear-fractional deformation depending on $\theta$ to the three-circle configuration, and the corresponding transformation to $Q$ (this does not change the modulus of $Q$ which is a conformal invariant) we can get in the limit a non-geodesic circular rectangle shown in Fig. 6 (right). Thus the modulus of $Q$ tends to a finite positive value when $\theta \rightarrow 1$. Computation in Eremenko and Gabrielov (2015) shows that this value is $K \approx 0.630963$.

Theorem 4.2 Let $\Gamma$ be one of the nets described in Theorem 3.1, and $\theta \in(0,1)$. Then the modulus of $Q(\Gamma, \theta)$ tends to 0 when $\theta \rightarrow 0$, and to a finite positive value when $\theta \rightarrow 1$.

Proof When $\theta \rightarrow 0$, none of the arcs connecting $a_{1}$ and $a_{3}$ contract, while the triangle $T_{\mu}$ (resp., $T_{\nu}$ ) contains either a short arc of $\Gamma$ or a side $L_{4}$ (resp., $L_{2}$ ) of $Q$ that maps to either the $\operatorname{arc} M R$ or the $\operatorname{arc} N P$ of $C$, connecting its apex $a_{3}$ (resp., $a_{1}$ ) with a point $p \neq a_{1}$ (resp., $p \neq a_{3}$ ) on its base. These two arcs contract to points when $\theta \rightarrow 0$. Hence the distance between the sides $L_{1}$ and $L_{3}$ of $Q$ tends to 0 when $\theta \rightarrow 0$. At the same time, there are no short arcs of $\Gamma$ having one end on $L_{2}$ and another end on $L_{4}$, other than those connecting $a_{1}$ and $a_{3}$ which do not contract as $\theta \rightarrow 0$. Thus the distance between the sides $L_{2}$ and $L_{4}$ does not tend to 0 as $\theta \rightarrow 0$. This implies that the modulus of $Q$ tends to 0 as $\theta \rightarrow 0$ (see Eremenko et al. 2016a, Section 15). 

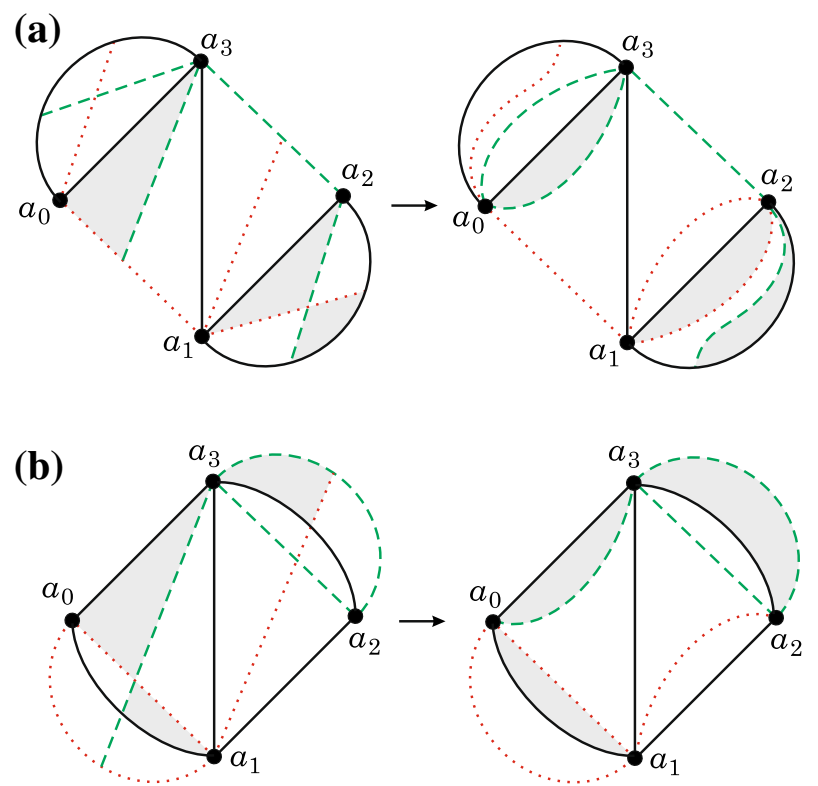

Fig. 7 Deformation of spherical rectangles with the angles $\frac{3}{2}, \frac{5}{2}, \frac{3}{2}, \frac{5}{2}$
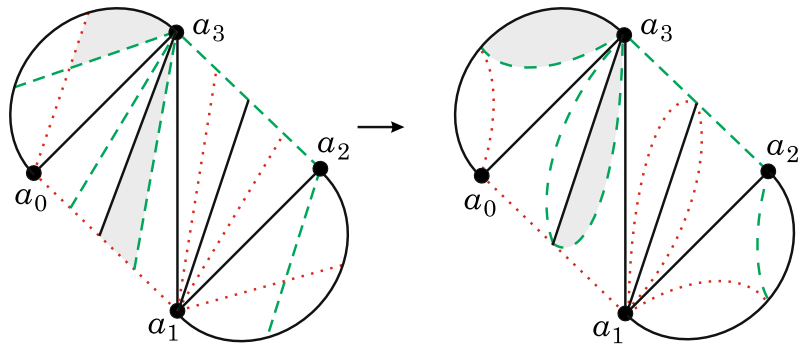

Fig. 8 Deformation of spherical rectangles with the angles $\frac{3}{2}, \frac{7}{2}, \frac{3}{2}, \frac{7}{2}$

When $\theta \rightarrow 1$, the short arcs connecting $a_{1}$ and $a_{3}$ contract, thus both the distance between $L_{1}$ and $L_{3}$ and the distance between $L_{2}$ and $L_{4}$ tend to 0 . To understand the limit of the modulus of $Q$, we apply a linear-fractional transformation depending on $\theta$ as in Example 4.1 to the three-circle configuration, so that the short arc $M N$ of $C$ contracts, while the short arc $P R$ does not. In the non-geodesic limit (see Fig. 5c) the circles $C^{\prime}$ and $C^{\prime \prime}$ become tangent (when their tangency point is mapped to $\infty$ as in Fig. 5d, they become parallel lines). All short arcs of $\Gamma$ connecting $a_{1}$ and $a_{3}$ map to $P R$, and all arcs of $C$ connecting the apex of a triangle $T_{\mu}$ (resp., $T_{\nu}$ ) with a point on its base, map to either $M R$ or $N P$. Since neither $M R$ nor $N P$ contracts when $\theta \rightarrow 1$, and $P R$ does not contract after the linear-fractional transformation, the distances between opposite sides of $Q$ do not tend to 0 in the limit. Thus $Q$ converges to a non-geodesic circular rectangle (see Figs. 6, 7, 8,9) and the modulus of $Q$ tends to a finite positive value. 


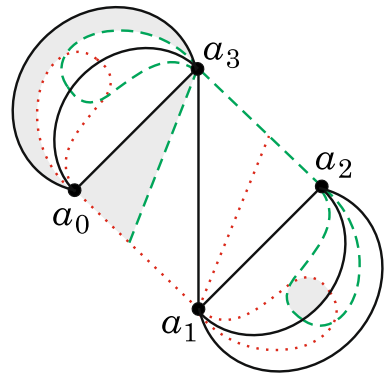

(p)

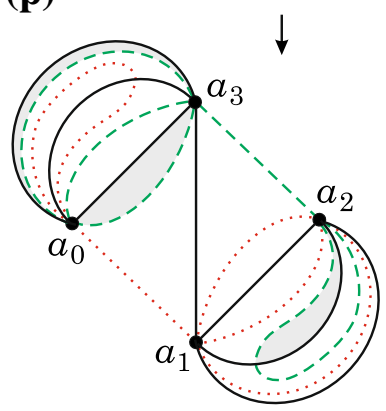

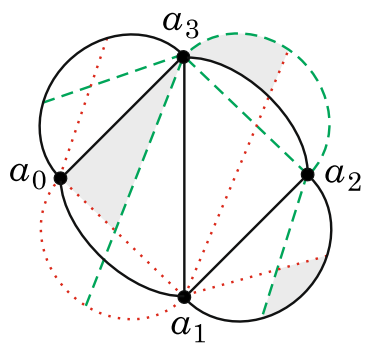

(q)

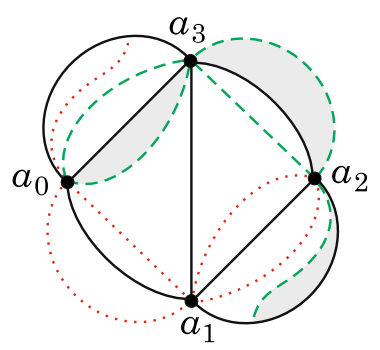

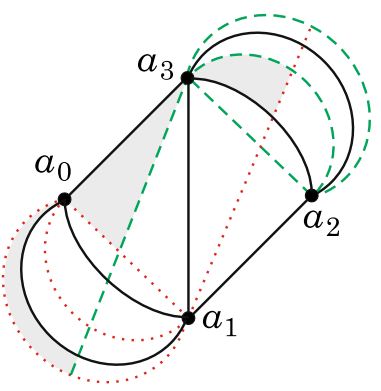

(r)

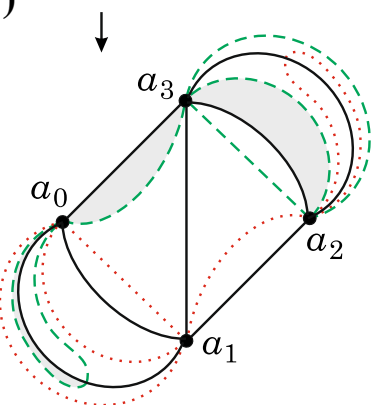

Fig. 9 Three combinatorially distinct nets of spherical rectangles with the angles $\frac{5}{2}, \frac{7}{2}, \frac{5}{2}, \frac{7}{2}$, and their deformations

Remarks on computation of limit moduli $K$ The boundary of the limit rectangle described in the proof of Theorem 4.2 is mapped by developing map into three straight lines (see Fig. 5d). This allows to represent the developing map by the SchwarzChristoffel formula. Condition that the points $P$ and $Q$ are on the same vertical line imposes one real equation which permits to determine the modulus of the rectangle $K$. See Eremenko and Gabrielov (2015) where the simplest example is described in all detail. The number of solutions to this equation is the number of nets with given angles. To determine which solution corresponds to which net, we use the evident inequalities between the moduli of degenerate rectangles (shown in the right of Figs. 7, 8, and in the bottom of Fig. 9).

Example 4.3 Two combinatorially distinct nets of spherical rectangles with the angles $(3 / 2,5 / 2,3 / 2,5 / 2)$, and the nets of their non-geodesic limits when $\theta \rightarrow 1$, are shown in Fig. 7a, b. The moduli $K_{a}$ and $K_{b}$ of the limiting rectangles are $K_{a} \approx 0.5433144$ and $K_{b} \approx 1.193606$, respectively. Figure 10 shows schematically the areas of existence of these spherical rectangles (Nets $a$ and $b$ ) and their involution-symmetric rectangles (Nets $\mathrm{a}^{\prime}$ and $\mathrm{b}^{\prime}$ ) for different values of the modulus $K$.

Example 4.4 Three combinatorially distinct nets of spherical rectangles with the angles $(5 / 2,7 / 2,5 / 2,7 / 2)$, and the nets of their non-geodesic limits when $\theta \rightarrow 1$, are shown in Fig. 9p, q, r. The moduli $K_{p}, K_{q}, K_{r}$ of the limiting rectangles are $K_{p} \approx 0.476966, K_{q} \approx 0.887943, K_{r} \approx 1.458956$, respectively. Figure 11 shows schematically the areas of existence of these spherical rectangles (Nets p, q, r) and 


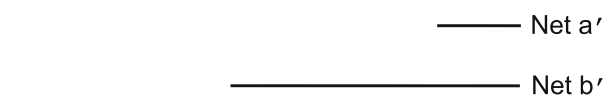

Net $b$

Net a

$$
0 \quad K_{\mathrm{a}} \quad 1 / K_{\mathrm{b}} \quad 1 \quad K_{\mathrm{b}} \quad 1 / K_{\mathrm{a}} \quad \infty
$$

Fig. 10 Existence of spherical rectangles with the angles $\frac{3}{2}, \frac{5}{2}, \frac{3}{2}, \frac{5}{2}$

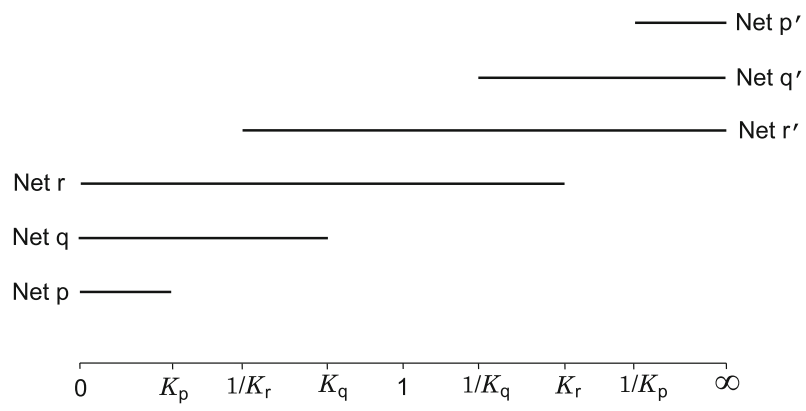

Fig. 11 Existence of spherical rectangles with the angles $\frac{5}{2}, \frac{7}{2}, \frac{5}{2}, \frac{7}{2}$

their involution-symmetric rectangles $\left(\right.$ Nets $\left.\mathrm{p}^{\prime}, \mathrm{q}^{\prime}, \mathrm{r}^{\prime}\right)$ for different values of the modulus $K$.

Example 4.5 A net of a spherical rectangle with the angles $(3 / 2,7 / 2,3 / 2,7 / 2)$, and the net of its non-geodesic limit when $\theta \rightarrow 1$, is shown in Fig. 8. The modulus $K$ of the limiting rectangle is $K \approx 0.4173$.

Remarks and conjectures Each of the three nets p, q, r in Fig. 11 produces a continuous family where the modulus $K$ can be arbitrarily small. So for sufficiently small $K$ there are at least three different marked spherical quadrilaterals with the angles $(5 / 2,7 / 2,5 / 2,7 / 2)$. Similarly, one can conclude from Fig. 11 that there are at least two quadrilaterals with modulus $K \in\left(K_{\mathrm{p}}, 1 / K_{\mathrm{r}}\right.$ at least three for $K \in\left(1 / K_{\mathrm{r}}, K_{\mathrm{q}}\right)$, at least two for $K \in\left(K_{\mathrm{q}}, 1 / K_{\mathrm{q}}\right)$, at least three for $K \in\left(1 / K_{\mathrm{q}}, K_{\mathrm{r}}\right)$, at least two for $\left.K \in(K) \mathrm{r}, 1 / K_{\mathrm{p}}\right)$, and at least three for $K>1 / K_{\mathrm{p}}$.

Similar conclusions apply to Fig. 10. Our computations show that in fact these lower estimates are equalities, in all cases which we computed. Actually $K$ is a monotone function of $\theta$ in all these cases, but we do not expect this monotonicity to hold for all angles.

So Theorem 1.5 gives only lower estimates for the number of quadrilaterals with given angles and modulus, when this modulus is small or large. This lower estimate is $N$ or $M_{j}$ (see (1.11), (1.12), (1.13). We conjecture that these lower estimates are exact, and that we have equality for large and small moduli. All computed examples confirm this. 


\section{Counting nets of spherical rectangles with given angles}

We want to answer the following question: Given four non-negative integers $A_{0}, \ldots, A_{3}$, how many nets of marked spherical rectangles with the integer parts $A_{0}, \ldots, A_{3}$ of the angles at their corners $a_{0}, \ldots, a_{3}$ do exist?

It is enough to answer this question for the spherical rectangles of the first type (satisfying Assumption 1), since all rectangles of the second type can be obtained then by an involution preserving the marked corner. Also, we may assume that $A_{0}+A_{2}+2 \leq$ $A_{1}+A_{3}$, which is true for the marked spherical rectangles satisfying Assumption 2 (see Remark 3.2). The answer for the rectangles with $A_{1}+A_{3}+2 \leq A_{0}+A_{2}$, having an arc connecting corners $a_{0}$ and $a_{2}$, can be obtained then by a different choice of the initial corner.

We start with listing operations on the nets which do not change the angles of a spherical rectangle $Q$. Assuming notation of Theorem 3.1 and Remark 3.2, we have expressions (3.1) for the angles of $Q$.

Operation I If $\kappa>0, i \geq 2, l=0, \mu=0$ then

$$
\kappa \mapsto \kappa-1, \quad v \mapsto v+4, \quad i \mapsto i-2, \quad m \mapsto m+2
$$

The inverse operation is possible when $l=0, m \geq 2, \mu=0, v \geq 4$.

Operation II If $\kappa>0, i=0, l \geq 2, v=0$ then

$$
\kappa \mapsto \kappa-1, \quad \mu \mapsto \mu+4, \quad k \mapsto k+2, \quad l \mapsto l-2
$$

The inverse operation is possible when $i=0, k \geq 2, \mu \geq 4, v=0$.

Operation III If $\kappa>0, i=1, l=0, \mu=0$ then

$$
\kappa \mapsto \kappa-1, \quad \mu \mapsto 1, \quad v \mapsto v+3, \quad i \mapsto 0, m \mapsto m+1
$$

The inverse operation is possible when $i=l=0, m \geq 1, \mu=1, v \geq 3$.

Operation IV If $\kappa>0, i=0, l=1, v=0$ then

$$
\kappa \mapsto \kappa-1, \quad \mu \mapsto \mu+3, \quad v \mapsto 1, \quad k \mapsto k+1, \quad l \mapsto 0
$$

The inverse operation is possible when $i=l=0, k \geq 1, \mu \geq 3, v=1$.

Operation V If $\kappa>0, i=l=0$ then

$$
\kappa \mapsto \kappa-1, \quad \mu \mapsto \mu+2, \quad \nu \mapsto v+2
$$

The inverse operation is possible when $i=l=0, \mu \geq 2, v \geq 2$.

Operation VI If $i>0, l>0, \mu=v=0$ then

$$
i \mapsto i-1, \quad k \mapsto k+1, \quad l \mapsto l-1, \quad m \mapsto m+1
$$


The inverse operation is possible when $k>0, m>0, \mu=v=0$.

Lemma 5.1 Let $Q$ be a marked spherical rectangle satisfying Assumptions 1 and 2 .

(a) If $\kappa>0$ and $\min (i, l)=0$ then there is a unique operation among Operations $I$-V that is applicable to $Q$ and results in a rectangle with the same angles as $Q$, with $\kappa$ reduced by 1.

(b) If $\min (i, l)=0$ then at most one operation among inverses to Operations $I-V$ is applicable to $Q$. If $\min (i, l)>0$ then neither Operations $I$ - $V$ nor their inverses are applicable to $Q$.

(c) If $\min (i, l)>0$, then iteration of Operation VI applied to $Q$ results in a rectangle with the same angles as $Q$, same $\kappa, \mu, v$, and $\min (i, l)=0$.

Proof We start with a proof of (a), assuming $\kappa>0$. If $i=l=0$ then Operation $\mathrm{V}$ is applicable. If $i=1, l=0$ then $\mu=0$ and Operation III is applicable. If $i \geq 2, l=0$ then $\mu=0$ and Operation I is applicable. If $i=0, l=1$ then $v=0$ and Operation IV is applicable. If $i=0, l \geq 2$ then $v=0$ and Operation II is applicable. It is easy to check that only one of the operations $\mathrm{I}-\mathrm{V}$ is applicable in each of these cases.

To prove (b) note first that inverses to Operations I-V are only possible when $\min (i, l)=0$. Next, for given $\mu$ and $v$, conditions on $\mu$ and $v$ for applicability of the operations inverse to Operations I-V may hold for at most one of these operations.

If $\min (i, l)>0$ then Operation VI reduces $i, l$ and $\min (i, l)$ by 1 , and does not change $\kappa, \mu, v$, which proves (c).

Corollary 5.2 For given $A_{0}, \ldots, A_{3}$, the set of values of $\kappa$ that may appear in the nets of marked spherical rectangles of the first type with $\delta \geq 1$ is either empty (in which case spherical rectangles with such angles do not exist) or an interval [0, $\left.\kappa_{\max }\right]$, for some integer $\kappa_{\max } \geq 0$ depending on $A_{0}, \ldots, A_{3}$. In the latter case, there are exactly $\kappa_{\max }+1$ combinatorially distinct nets of marked spherical rectangles of the first type with given $A_{0}, \ldots, A_{3}$ and $\min (i, l)=0$.

For a marked spherical rectangle $Q$ satisfying Assumption 2, define

$$
\delta=\left(A_{1}+A_{3}-A_{0}-A_{2}\right) / 2=2 \kappa+1+(\mu+v) / 2 .
$$

Then $\delta \geq 1$ is either integer or half-integer.

Lemma 5.3 Let $Q$ be a marked spherical rectangle with $\min (i, l)=0$ satisfying Assumptions 1 and 2. The net of $Q$ cannot be obtained from a net of some other spherical rectangle by one of the operations inverse to Operations $I-V$ if and only if one of the following twelve conditions is satisfied:

(a) $\mu=v=0$;

(b) $\mu=0, v=1$;

(c) $\mu=1, v=0$;

(d) $\mu=v=1$;

(e) $\mu=0, v=2$;

(f) $\mu=2, v=0$;

(g) $\mu=0, v=3$; 
(h) $\mu=3, v=0$;

(i) $\mu=1, v \geq 3, m=0$;

(j) $\mu \geq 3, v=1, k=0$;

(k) $\mu=0, v \geq 4, m \leq 1$;

(l) $\mu \geq 4, v=0, k \leq 1$.

For given $A_{0}, \ldots, A_{3}$ with $A_{1}+A_{3} \geq A_{0}+A_{2}+2$, a net of a marked spherical rectangle with $\min (i, l)=0$ satisfying Assumptions 1 and 2 may satisfy at most one of these conditions.

At most one (up to combinatorial equivalence) net of a marked spherical rectangle $Q$ satisfying Assumptions 1 and 2 , with given $A_{0}, \ldots, A_{3}$ and $\min (i, l)=0$, may satisfy any of these conditions. The value of $\kappa$ for such rectangle $Q$ is

$$
\left[\min \left(\frac{A_{1}-1}{2}, \frac{A_{3}-1}{2}, \frac{\delta-1}{2}\right)\right] .
$$

Proof One can easily check case by case that none of the operations inverse to Operations I-V can be applied if and only if one of the conditions (a)-(1) is satisfied. Note that it is enough to assume $\mu \leq v$, and to check that with this assumption none of the operations inverse to Operations I,III, V can be applied if and only if one of the conditions (a), (b), (d), (e), (g), (i), (k) is satisfied. The case $\mu \geq v$ follows by rotation of the net exchanging $a_{0}$ with $a_{2}, a_{1}$ with $a_{3}, \mu$ with $v, i$ with $l$, and $k$ with $m$.

Let now $Q$ be a marked spherical rectangle satisfying Assumptions 1 and 2, with given $A_{0}, \ldots, A_{3}, A_{1}+A_{3} \geq A_{0}+A_{2}+2, \min (i, l)=0$, and $\mu \leq v$.

If $Q$ satisfies (a) then $\delta=2 \kappa+1$ is an odd integer, $A_{1} \geq \delta, A_{3} \geq \delta$. If, in addition, $l=0$ then $A_{2}=k$ and $A_{3}=\delta+m$, thus $i=A_{0}-m=A_{0}-A_{3}+\delta=$ $\left(A_{0}+A_{1}-A_{2}-A_{3}\right) / 2$, and the net of $Q$ is completely determined by its angles. Such a net exists when $i \geq 0$ thus $A_{0}+A_{1} \geq A_{2}+A_{3}$. The case when $i=0$ is treated similarly, and a net with $i=0$ satisfying (a) exists when $A_{0}+A_{1} \leq A_{2}+A_{3}$. The net with $i=l=0$ satisfying (a) exists when $A_{0}+A_{1}=A_{2}+A_{3}$.

If $Q$ satisfies (b) then $\delta=2 \kappa+\frac{3}{2}$ thus $2 \delta \equiv 3 \bmod 4, A_{1} \geq \delta+\frac{1}{2}, A_{3} \geq \delta-\frac{1}{2}$, $l=0, A_{2}=k, A_{3}=m+\delta-\frac{1}{2}$ thus $m=A_{3}-\delta+\frac{1}{2}, A_{0}=i+m=i+A_{3}-\delta+\frac{1}{2}$ thus $i=A_{0}-A_{3}+\delta-\frac{1}{2}=\left(A_{0}+A_{1}-A_{2}-A_{3}-1\right) / 2$. A net satisfying (b) is completely determined by its angles. It exists when $i \geq 0$, thus $A_{0}+A_{1} \geq A_{2}+A_{3}+1$. Similarly, if $Q$ satisfies (c) then $\delta=2 \kappa+\frac{3}{2}$, and its net is completely determined by its angles. It exists when $A_{1} \geq \delta-\frac{1}{2}, A_{3} \geq \delta+\frac{1}{2}, A_{2}+A_{3} \geq A_{0}+A_{1}+1$.

If $Q$ satisfies (d) then $\delta=A_{3}-A_{0}=A_{1}-A_{2}=2 \kappa+2$ is a positive even integer, $i=l=0$. Then $A_{0}=m, A_{2}=k$, and the net is completely determined by its angles. It exists when $A_{0}+A_{1}=A_{2}+A_{3}$.

If $Q$ satisfies (e) then $\delta=2 \kappa+2$ is a positive even integer, $A_{1} \geq \delta+1, A_{3} \geq \delta-1$, $l=0, A_{0}=i+m, A_{2}=k, A_{1}=i+k+\delta+1$ thus $i=A_{1}-A_{2}-\delta-1=$ $\left(A_{0}+A_{1}-A_{2}-A_{3}-2\right) / 2, m=A_{3}-\delta+1$, and the net is completely determined by its angles. It exists when $i \geq 0$ thus $A_{0}+A_{1} \geq A_{2}+A_{3}+2$. Similarly, if $Q$ satisfies (f) then $\delta=2 \kappa+2$, and its net is completely determined by its angles. It exists when $A_{1} \geq \delta-1, A_{3} \geq \delta+1, A_{2}+A_{3} \geq A_{0}+A_{1}+2$. 
If $Q$ satisfies (g) then $\delta=2 \kappa+\frac{5}{2}$ thus $2 \delta \equiv 1 \bmod 4, A_{1} \geq \delta+\frac{3}{2}, A_{3} \geq \delta-\frac{3}{2}$, $l=0, A_{2}=k, A_{3}=m+\delta-\frac{3}{2}$ thus $m=A_{3}-\delta+\frac{3}{2}, A_{0}=i+m=i+A_{3}-\delta+\frac{3}{2}$ thus $i=A_{0}-A_{3}+\delta-\frac{3}{2}=\left(A_{0}+A_{1}-A_{2}-A_{3}-3\right) / 2$. A net satisfying $(\mathrm{g})$ is completely determined by its angles. It exists when $i \geq 0$, thus $A_{0}+A_{1} \geq A_{2}+A_{3}+3$. Similarly, if $Q$ satisfies (h) then $\delta=2 \kappa+\frac{5}{2}$, and its net is completely determined by its angles. It exists when $A_{1} \geq \delta+\frac{3}{2}, A_{3} \geq \delta-\frac{3}{2}, A_{2}+A_{3} \geq A_{0}+A_{1}+3$.

If $Q$ satisfies either (i), (j), (k) or (l) then $\delta=2 \kappa+3$ is an odd integer, $\delta \geq 3$. If $Q$ satisfies (i) then $A_{3}=\delta-1, i=l=0, A_{0}=0, A_{2}=k$. If $Q$ satisfies (j) then $A_{1}=\delta-1, i=l=0, A_{0}=m, A_{2}=0$. If $Q$ satisfies (k) then $A_{3}=\delta-2+m$, $l=0, A_{0}=i+m, A_{2}=k$. A net satisfying (k) exists when $i=A_{0}-m=$ $A_{0}-A_{3}+\delta-2=\left(A_{0}+A_{1}-A_{2}-A_{3}-4\right) / 2 \geq 0$, thus $A_{0}+A_{1} \geq A_{2}+A_{3}+4$. If $Q$ satisfies (1) then $A_{1}=\delta-2+k, i=0, A_{0}=m, A_{2}=k+l$. A net satisfying (l) exists when $l=A_{2}-k=A_{2}-A_{1}+\delta-2=\left(A_{2}+A_{3}-A_{0}-A_{1}-4\right) / 2 \geq 0$, thus $A_{2}+A_{3} \geq A_{0}+A_{1}+4$. A net satisfying either (i), (j), (k) or (l) is completely determined by its angles.

Lemma 5.4 A marked spherical rectangle $Q$ satisfying Assumptions 1 and 2, with given $A_{0}, \ldots, A_{3}$ and $\min (i, l)=0$ exists if and only if

$$
\min \left(A_{1}, A_{3}, \delta\right) \geq 1
$$

and there are exactly

$$
\left[\min \left(\frac{A_{1}+1}{2}, \frac{A_{3}+1}{2}, \frac{\delta+1}{2}\right)\right]
$$

combinatorially distinct nets of such spherical rectangles.

Proof It follows from Corollary 5.2 and Lemma 5.3 that existence of a rectangle $Q$ satisfying conditions of Lemma 5.4 implies that the number in (5.2) is non-negative, which is equivalent to (5.3), and that the number of combinatorially distinct nets of such rectangles in that case is the number in (5.2) plus 1 , which is the number in (5.4). Thus we have only to prove that for any $A_{0}, \ldots, A_{3}$ satisfying (5.3) there exists a rectangle $Q$ satisfying conditions of Lemma 5.4.

It follows from Corollary 5.2 that, if a rectangle with given $A_{0}, \ldots, A_{3}$ satisfying conditions of Lemma 5.4 exists, there exists also a rectangle with $\kappa=0$ with the same angles satisfying the same conditions. We are going to construct a net of such a rectangle for any $A_{0}, \ldots, A_{3}$ satisfying (5.3).

There are three possible cases: (i) $A_{1}>A_{2}$ and $A_{3}>A_{0}$; (ii) $A_{1}>A_{2}$ and $A_{3} \leq A_{0}$; (iii) $A_{1} \leq A_{2}$ and $A_{3}>A_{0}$. Note that $A_{1} \leq A_{2}$ and $A_{3} \leq A_{0}$ is not possible because $\delta=\left(A_{1}+A_{3}-A_{0}-A_{2}\right) / 2 \geq 1$.

In case (i) let $i=l=0, m=A_{0}, k=A_{2}, \mu=A_{3}-m-1=A_{3}-A_{0}-1$, $v=A_{1}-k-1=A_{1}-A_{2}-1$.

In case (ii) let $\mu=l=0, m=A_{3}-1, k=A_{2}, i=A_{0}-m=A_{0}-A_{3}+1$, $v=A_{1}-i-k-1=A_{1}+A_{3}-A_{0}-A_{2}-2=2(\delta-1)$. 
In case (iii) let $v=i=0, k=A_{1}-1, m=A_{0}, l=A_{2}-k=A_{2}-A_{1}+1$, $\mu=A_{3}-l-m-1=A_{1}+A_{3}-A_{0}-A_{2}-2=2(\delta-1)$.

This completes the proof of Lemma 5.4.

Definition 5.5 A marked spherical rectangle is special if $\delta$ is an odd integer and either $A_{1} \geq \delta>0$ and $A_{3} \geq \delta>0$ or $A_{0} \geq-\delta>0$ and $A_{2} \geq-\delta>0$.

Lemma 5.6 A marked spherical rectangle $Q$ satisfying Assumptions 1 and 2 with given $A_{0}, \ldots, A_{3}$ is special if an only if there exists a rectangle with the same angles whose net has $\mu=v=0$. For given $A_{0}, \ldots, A_{3}$ satisfying conditions of Definition 5.5 with $\delta>0$, there are

$$
\min \left(A_{0}, A_{1}-\delta, A_{2}, A_{3}-\delta\right)
$$

special rectangles satisfying Assumptions 1 and 2 , with $\mu=v=0$ and $\min (i, l)>0$.

Proof If $\min (i, l)>0$ for the net of $Q$ then $\mu=v=0$. From Lemma 5.1 (c), iteration of Operation VI applied to $Q$ results in a unique rectangle $Q_{0}$ with the same angles as $Q, \mu=v=0$ and $\min (i, l)=0$. Thus $Q_{0}$ satisfies Lemma 5.3 (a). If $\min (i, l)=0$ for the net of $Q$ then, from Corollary 5.2, there is a unique rectangle with the same angles as $Q$ satisfying one of the conditions (a)-(1) of Lemma 5.3. One can easily check that $\delta$ is an odd integer, $A_{1} \geq \delta$ and $A_{3} \geq \delta$ only in case (a) of Lemma 5.3. Conversely, a rectangle satisfying condition (a) of Lemma 5.3 is clearly special, thus any rectangle $Q$ with the same angles is also special. Finally, the number in (5.5) is obtained by counting distinct rectangles that can be obtained from a rectangle with $\mu=v=0$ by iterating Operation VI and its inverse (compare with Lemma 11.2 in Eremenko et al. 2016a).

Theorem 5.7 Let $Q$ be a marked spherical rectangle satisfying Assumptions 1 and 2 , with given $A_{0}, \ldots, A_{3}$. If $Q$ is not special then there are (5.4) combinatorially distinct nets of marked spherical rectangles satisfying Assumptions 1 and 2 with the same angles as $Q$. If $Q$ is special then the number in $(5.4)$ is $(1+\delta) / 2$, and there are additionally (5.5) nets of marked spherical rectangles satisfying Assumptions 1 and 2 with the same angles as $Q$, thus the total number of combinatorially distinct nets is

$$
\min \left(A_{0}+\frac{1+\delta}{2}, A_{1}+\frac{1-\delta}{2}, A_{2}+\frac{1+\delta}{2}, A_{3}+\frac{1-\delta}{2}\right) .
$$

Proof This follows from Lemmas 5.4 and 5.6.

Proof of Theorems 1.3 and 1.5. Lemmas 5.4 and 5.6 imply that a marked spherical rectangle satisfying Assumptions 1 and 2 exists if and only if (5.3) holds. Since this condition is symmetric with respect to $A_{1}$ and $A_{3}$, it remains true for spherical rectangles of the second type satisfying $A_{0}+A_{2}<A_{1}+A_{3}$, as any such rectangle can be obtained from a rectangle satisfying Assumptions 1 and 2 by a reflection preserving $a_{0}$ and $a_{2}$, exchanging $a_{1}$ and $a_{3}$. 
If $Q$ is a marked spherical rectangle of either first of second type satisfying $A_{0}+$ $A_{2}>A_{1}+A_{3}$, replacing $a_{0}$ by $a_{1}$ as an initial corner, and relabeling the corners accordingly, results in a marked spherical rectangle $Q^{\prime}$ of either second or first type, with the integer parts of the angles $\left(A_{0}^{\prime}, A_{1}^{\prime}, A_{2}^{\prime}, A_{3}^{\prime}\right)=\left(A_{1}, A_{2}, A_{3}, A_{0}\right)$ and $\delta^{\prime}=$ $\left(A_{1}^{\prime}+A_{3}^{\prime}-A_{0}^{\prime}-A_{2}^{\prime}\right) / 2=-\delta$. Applying the above arguments to $Q^{\prime}$ we see that a marked spherical rectangle $Q$ with $A_{0}+A_{2}>A_{1}+A_{3}$ exists if and only if

$$
\min \left(A_{0}, A_{2},-\delta\right) \geq 1
$$

Combining (5.3) and (5.7) we get the statement of Theorem 1.3. The statement of Theorem 1.5 follows from (5.4) and (5.6) applied to either $Q$ or $Q^{\prime}$ in a similar way.

Lemma 5.8 Let $Q$ be a marked spherical rectangle with the angles satisfying $A_{0}=$ $A_{2}, A_{1}=A_{3}$. Then there is an orientation-preserving isometry $\rho: Q \rightarrow Q$ such that $\rho\left(a_{0}\right)=a_{2}$ and $\rho\left(a_{1}\right)=a_{3}$.

Proof Due to Proposition 1.2, it is enough to prove that the net $\Gamma$ of $Q$ is symmetric with respect to a transformation exchanging $a_{0}$ with $a_{2}$ and $a_{1}$ with $a_{3}$, and that this symmetry of $\Gamma$ maps any short arc of $\Gamma$ connecting $a_{1}$ with $a_{3}$ is mapped to a (possibly, different) short arc connecting $a_{1}$ with $a_{3}$. To show this, we have only to check (assuming that $Q$ satisfies Assumptions 1 and 2) that $\mu=v, i=l$, and $k=m$ in the notations of Theorem 3.1.

Suppose first that $\mu=v=0$. Then $A_{0}=A_{2}$ implies $i+m=k+l$, and $A_{1}=A_{3}$ implies $i+k=m+l$ (see (3.1) in Remark 3.2). Adding up these two equalities yields $i=l$, and subtracting them yields $k=m$.

If $\mu>0$ and $v>0$ then $i=l=0$, thus $A_{0}=m, A_{2}=k, A_{1}=k+1+2 \kappa+v$, and $A_{3}=m+1+2 \kappa+\mu$. Since $A_{0}=A_{2}$, we have $k=m$, then $A_{1}=A_{3}$ yields $\mu=\nu$.

If $\mu>0$ but $v=0$ then $i=0$, thus $A_{0}=m, A_{1}=k+1+2 \kappa, A_{2}=k+l$, and $A_{3}=l+m+1+2 \kappa+\mu$. Since $A_{0}=A_{2}$, we have $m=k+l$, thus $A_{3}=$ $k+2 l+1+2 \kappa+\mu>A_{1}$, a contradiction. Similarly, $\mu=0$ and $v>0$ is not possible. This completes the proof.

Theorem 5.9 A spherical rectangle $Q$ with the angles at two of its opposite corners equal $\alpha$, and the angles at two other opposite corners equal $\beta$, exists if and only if $|\beta-\alpha| \geq 1$. If $\beta-\alpha$ is even then there are $|\beta-\alpha|$ non-isometric spherical rectangles with these angles, $|\beta-\alpha| / 2$ of them satisfying Assumption 1. If $\beta-\alpha$ is odd then there are $\alpha+\beta$ non-isometric spherical rectangles with these angles, $(\alpha+\beta) / 2$ of them satisfying Assumption 1.

This follows from Lemma 5.8 and Theorems 1.3 and 1.5.

Example 5.10 The net in Fig. 6 is special, with $A_{0}=A_{2}=0, A_{1}=A_{3}=1, \delta=1$. According to (5.6) and Theorem 5.9, there is a unique net of a marked spherical rectangle of the first type with these angles.

The two nets in Fig. 7 are special with $A_{0}=A_{2}=1, A_{1}=A_{3}=2, \delta=1$. According to (5.6) and Theorem 5.9, there are two nets of marked spherical rectangles of the first type with these angles. 
The net in Fig. 8 is not special, with $A_{0}=A_{2}=1, A_{1}=A_{3}=3, \delta=2$. According to (5.4) and Theorem 5.9, there is a unique net of a marked spherical rectangle of the first type with these angles.

The three nets in Fig. 9 are special with $A_{0}=A_{2}=2, A_{1}=A_{3}=3, \delta=1$. According to (5.6) and Theorem 5.9, there are three nets of marked spherical rectangles of the first type with these angles.

\section{References}

Chai, C.-L., Lin, C.-S., Wang, C.-L.: Mean field equations, hyperelliptic curves and modular forms: I. arXiv: 1502.03297

Darboux, G.: Sur une équation linéaire. C. R. Acad. Sci. Paris Trans. 94, 1645-1648 (1882)

Eremenko, A., Gabrielov, A., Tarasov, V.: Metrics with conic singularities and spherical polygons. Ill. J. Math. 58, 739-755 (2014)

Eremenko, A., Gabrielov, A., Tarasov, V.: Metrics with four conic singularities and spherical quadrilaterals. Conformal Geom. Dyn. 20, 128-175 (2016a)

Eremenko, A., Gabrielov, A., Tarasov, V.: Spherical quadrilaterals with three non-integer angles. J. Math. Phys. Anal. Geom. 12, 134-167 (2016b)

Eremenko, A., Gabrielov, A.: On metrics of curvature 1 with four conic singularities on tori and on the sphere. arXiv: 1508.06510

Eremenko, A.: Metrics of positive curvature with conic singularities on the sphere. Proc. Am. Math. Soc. 132, 3349-3355 (2004)

Eremenko, A., Gabrielov, A., Shapiro, M., Vainshtein, A.: Rational functions and real Schubert calculus. Proc. AMS 134(4), 949-957 (2006)

Hermite, C.: Sur l'équation de Lamé, Extrait de feuilles authographiées du Course d'Analyse de l'École Polytechnique, $1^{\text {re }}$ Division, 1872-1873, $32^{e}$ leçon. Oeuvres, t. III, p. 118-122. Paris, Gauthier-Villars (1912)

Khovanskii, A.: On a class of systems of transcendental equations. Soviet Math. Dokl. 22, 762-765 (1980)

Klein, F.: Vorlesungen über das Insider und die Auf̈̈nug der Clonking Von föften Grade. Birkhäuser Verlag, Basel (1993)

Lin, C.-S., Wang, C.-L.: Elliptic functions Green functions and the mean field equations on tori. Ann. Math. 172, 911-954 (2010)

Luo, F., Tian, G.: Liouville equation and spherical convex polytopes. Proc. Am. Math. Soc. 116(4), 11191129 (1992)

Mondello, G., Panov, D.: Spherical metrics with conical singularities on a 2-sphere: angle constraints. Int. Math. Res. Not. 16, 4937-4995 (2016)

Schneps, L.: Dessins d'enfants on the Riemann sphere. In: The Grothendieck Theory of dessins d'enfants (Luminy, 1993), pp. 47-77, Cambridge Univ. Press, Cambridge (1994)

Speissegger, P.: The Pfaffian closure of an o-minimal structure. J. für die reine und angew. Math. 508, 189-211 (1999)

Troyanov, M.: Prescribing curvature on compact surfaces with conical singularities. Trans. Am. Math. Soc. 324, 793-821 (1991)

van den Dries, L.: Tame topology and o-minimal structures. London Math. Soc. Lecture Notes Series 248, Cambridge University Press, Cambridge (1998)

Van Vleck, E.: A determination of the number of real and imaginary roots of the hypergeometric series. Trans. Am. Math. Soc. 3, 110-131 (1902)

Wilkie, A.J.: A theorem of the complement and some new o-minimal structures. Selecta Math. (N.S.). 5, 397-421 (1999) 